Negative Cancer Beliefs: Socioeconomic Differences from the Awareness and Beliefs about

\title{
Cancer Survey
}

Running title: Negative cancer beliefs and socioeconomic status

Elizabeth A. Sarma, PhD, MPH, ${ }^{1,2^{*}}$ Samantha L. Quaife, $\mathrm{PhD},{ }^{3}$ Katharine A. Rendle, MSW, $\mathrm{PhD}, \mathrm{MPH},{ }^{4}$ Sarah C. Kobrin, $\mathrm{PhD}^{1}$

${ }^{1}$ Healthcare Delivery Research Program, Division of Cancer Control and Population Sciences, National Cancer Institute, Bethesda, MD, USA

${ }^{2}$ Cancer Prevention Fellowship Program, Division of Cancer Prevention, National Cancer Institute, Bethesda, MD, USA

${ }^{3}$ Department of Behavioral Science and Health, University College London, London, England

${ }^{4}$ Department of Family Medicine and Community Health, Perelman School of Medicine, University of Pennsylvania, Philadelphia, PA, USA

*Corresponding author: Elizabeth A. Sarma, Healthcare Delivery Research Program, Division of Cancer Control and Population Sciences, National Cancer Institute, 9609 Medical Center Dr., MSC 9761, Rockville, MD 20850 USA; Tel: (240) 276-7434, E-mail: elizabeth.sarma@nih.gov 


\begin{abstract}
Objective: Socioeconomic gaps in cancer mortality may be driven partially by poorer uptake of early detection behaviors among lower socioeconomic status (SES) groups. Lower SES groups may hold both fewer positive and more negative cancer beliefs that discourage these behaviors. We examined SES differences in positive and negative cancer beliefs in US adults.

Methods: We conducted telephone interviews with a population-representative sample, aged 50+, using the Awareness and Beliefs about Cancer $(\mathrm{ABC})$ instrument $(N=1,425)$. Cancer beliefs were measured using three positively and three negatively framed items. We used multivariable logistic regression models to examine associations between beliefs and education, which served as a marker of individual-level SES.
\end{abstract}

Results: Agreement with positive statements was high (>80\%) and did not vary with education, while agreement with negative statements varied. Relative to adults with a bachelor's degree, adults with a high school degree or less were more likely to agree that "treatment is worse than cancer" $(45.2 \%$ vs. $68.2 \%$; adjusted odds ratio $(\mathrm{aOR})=2.43,99 \%$ confidence interval $(\mathrm{CI})=1.50$ 3.94), cancer is "a death sentence" ( $17.4 \%$ vs. $33.2 \%$; aOR $=2.51,99 \% \mathrm{CI}=1.45-4.37)$, and they "would not want to know if I have cancer" (15.7\% vs. $31.6 \%$; aOR=2.88, 99\% $\mathrm{CI}=1.54-5.36)$. Conclusions: Positive cancer statements were generally endorsed, but negative statements were more frequently endorsed by lower SES groups. Additional work is needed to understand how negative beliefs develop and coexist alongside positive beliefs. Interventions to improve detection behaviors targeting lower SES groups may benefit from focusing on reducing negative beliefs, rather than increasing positive beliefs.

Keywords: Attitude; Belief; Cancer; Oncology; Education; Social Class; Psycho-Oncology 


\section{Background}

Socioeconomic inequalities in cancer mortality are widening in the US, with the largest gaps for the most preventable cancers. ${ }^{1}$ Lower survival has been attributed, in part, to later stage at diagnosis among lower socioeconomic status (SES) groups, which may be a result of lower engagement in early cancer detection behaviors. ${ }^{2}$ The Model of Pathways to Treatment describes the intervals an individual may move through before receiving treatment (appraisal, helpseeking, diagnostic, and pre-treatment), and the patient, provider, healthcare system, and disease factors that influence the length of these intervals. ${ }^{3}$ Delays can occur in any of these intervals to exacerbate SES inequalities. For example, the appraisal and help-seeking intervals, which occur prior to receiving a diagnosis and are collectively called the patient interval, may contribute to SES differences in cancer survival through differential engagement in early cancer detection behaviors. Early detection can be achieved through cancer screening and timely medical help seeking for potential cancer symptoms. ${ }^{4}$ SES disparities are well known within screening adults with lower education and income are less likely to have had a recent cervical, breast, or colorectal cancer screening. ${ }^{5}$ In addition, lower SES is associated with delayed help-seeking for potential cancer symptoms. ${ }^{6-9}$ In turn, lower engagement in these early detection behaviors may contribute to disparities in cancer mortality.

Research suggests that socioeconomic disparities in beliefs about cancer preventability and outcomes may help to explain the social gradient in early detection behavior. Indeed, negative beliefs about cancer have been associated with greater healthcare avoidance ${ }^{10}$ and lower cancer screening uptake. ${ }^{11}$ Greater endorsement of these beliefs among lower SES groups may contribute to cancer disparities through lower uptake of early detection behaviors. ${ }^{12,13}$ In support of this hypothesis, certain negative cancer beliefs have been more commonly reported among 
lower SES groups. For example, fatalistic attitudes (i.e., the belief that cancer risk is predetermined and cancer is fatal ${ }^{14}$ ) are more prevalent among lower SES groups. ${ }^{10,15-19}$

However, rather than holding solely negative beliefs as has frequently been described, research is beginning to suggest lower SES groups' attitudes towards cancer are more complex, comprising both positive and negative beliefs about different aspects of cancer at the same time. For example, using a population-based sample of adults in the UK, Quaife et al. ${ }^{20}$ examined SES differences in both positive and negative attitudes towards cancers using the Awareness and Beliefs about Cancer (ABC) survey. ${ }^{21}$ Results showed that though most adults, regardless of SES, endorsed the positive cancer beliefs, lower SES adults were significantly more likely to endorse negative cancer beliefs. In a similar analysis with data from the Danish version of the ABC survey, Hvidberg et al. ${ }^{22}$ also found that while most adults agreed with the positive statements about cancer, people with lower education were significantly more likely to agree with the negative statements.

To our knowledge, previous US studies examining SES differences in cancer beliefs have, to date, examined negative beliefs, and relied on items from the Health Information National Trends Survey (HINTS), which focuses on beliefs about cancer prevention. ${ }^{11,16,23-26}$ It is therefore unknown whether the results from the UK and Denmark are applicable to the US, which has a different healthcare system, and different recommendations for early detection and access to healthcare. The objective of the current study was to examine the associations of SES with both positive and negative cancer beliefs about outcomes and the value of early presentation in a population-based sample of US adults using data from the ABC survey. Determining whether these trends also exist in the US is important given that both positive and negative 
cancer beliefs may contribute to SES cancer disparities. In turn, results might be used to inform the design of interventions aimed to engage lower SES groups in early detection behaviors.

\section{Methods}

\section{Population}

The Awareness and Beliefs about Cancer (ABC) survey ${ }^{21}$ was developed as part of the International Cancer Benchmarking Partnership (ICBP) and was administered in the UK, Australia, Canada, Denmark, Sweden, and Norway in 2011 to investigate international differences in cancer outcomes. ${ }^{21,27}$ The US version was adapted with minor language changes but was nearly identical to the UK version. Using computer-assisted telephone interviews, the ABC survey was administered to a population-representative sample of English-speaking US adults aged 50 years and older $(N=1,425)$ from August to October 2014. Adults completed the survey through a landline $(n=1,325)$ or a cell phone $(n=100)$ interview. Survey design and nonresponse weights were developed to ensure that the study sample data were representative of the US population. Details of the sampling approach and weight development are described elsewhere. ${ }^{6}$ As this was a phone population-based survey, participants gave verbal informed consent. Ethical approval was obtained from the National Cancer Institute's Office of Human Subjects Research Participation (14CN046).

\section{Measures}

Six items assessed cancer beliefs, including three positively framed and three negatively framed (see Table 2 for positive and Table 3 for negative items). Participants were asked to indicate how much they agreed or disagreed with each item, with response categories of strongly disagree, tend to disagree, tend to agree, and strongly agree. In keeping with previous $\mathrm{ABC}$ 
studies, responses were dichotomized (strongly disagree/tend to disagree/don't know, or strongly agree/tend to agree), and refusals were coded as missing. ${ }^{20,22}$

In keeping with previous $\mathrm{ABC}$ studies, ${ }^{20,22}$ education was used as a proxy for individuallevel SES (bachelor's degree and above, some college, high school or lower). Information on sociodemographic factors, included gender (female or male), age (50-64y or 65-99y), marital status (married/cohabiting or single/divorced/widowed), and race (white or non-white). Selfrated health (good or fair/poor) and cancer experience (none or yes, self or friend/family member has been diagnosed with cancer) were also measured.

\section{Statistical Analysis}

Analyses were modeled after Quaife et al. ${ }^{20}$ to allow for comparison between the UK and US in endorsement of cancer beliefs and their associations with SES. Separate multivariable logistic regression models were used to assess the association between each cancer belief and SES, adjusting for sociodemographic factors, self-rated health, and cancer experience. Nonresponse and survey design weights were applied in the models. Statistical analyses were performed using Stata 16, and all tests were two-sided with a $p<.01$ significance level to account for multiple testing.

\section{Results}

In total, 1,425 adults participated in the survey. ${ }^{28}$ Eligibility was confirmed for 5,397 landline numbers. Of these, 1,839 households had at least one member aged 50 years or older, and 1,325 agreed to participate, while 462 refused and 52 only partially completed the survey (interview rate $=72.1 \%$ ). Eligibility was confirmed for 1,284 cell phone numbers. Of these, 156 individuals were aged 50 years or older, and 100 agreed to participate, while 44 refused and 12 only partially completed the survey (interview rate $=64.1 \%$ ). 
Table 1 shows characteristics of the weighted study sample. The average age of the sample was 63.5 years $(\mathrm{SD}=15.3)$. There were more women than men $(53.5 \%$ vs. $46.5 \%)$, adults aged 50-64 years than 65-99 years (56.0\% vs. $44.0 \%)$, adults who were married or cohabiting than single, divorced, or widowed (62.8\% vs. $37.2 \%)$, and White adults than nonWhite adults (79.2\% vs. $20.8 \%$ ). Approximately $30 \%$ of adults had acquired a bachelor's degree or above. Most adults reported that their health was good or very good (71.0\%) and had some experience with cancer $(82.5 \%)$.

In general, agreement with the positive cancer beliefs was high in the sample (Table 2). Most participants agreed that "people with cancer can expect to continue with normal activities and responsibilities" $(83.9 \%)$, that "cancer can often be cured" $(82.8 \%)$, and that "going to the doctor as quickly as possible after noticing a symptom of cancer could increase the chances of survival" (94.6\%). Table 2 shows associations between each positive cancer belief and SES, adjusting for sociodemographic factors, self-rated health, and cancer experience. In multivariable analyses, education was not significantly associated with any of the positive beliefs, nor were any of the other sociodemographic factors, except for gender. Participants who rated their health as fair or poor were significantly less likely to agree that "people with cancer can expect to continue with normal activities and responsibilities" $(75.0 \%$ vs. $87.8 \%$ in participants with good self-rated health), as were participants with cancer experience (91.6\% vs. $82.3 \%$ in participants with no cancer experience).

Agreement with the negative cancer beliefs showed relatively greater variability: $26.7 \%$ of participants agreed that "a diagnosis of cancer is a death sentence," $23.3 \%$ agreed that they "would not want to know if I have cancer," and 59.6\% agreed that "most cancer treatment is worse than the cancer itself" (Table 3). For each of the negative cancer beliefs, agreement varied 
markedly across education level: those with lower education were significantly more likely to agree with the statements. Compared with adults with a bachelor's degree or above, those with a high school degree or less were significantly more likely to agree that "a diagnosis of cancer is a death sentence" (17.4\% vs. 33.2\%, respectively), that they "would not want to know if I have cancer" $(15.7 \%$ vs. $31.6 \%)$, and that "most cancer treatment is worse than the cancer itself" (45.2\% vs. $68.2 \%)$. Few other variables showed associations with the negative cancer beliefs. Participants with cancer experience (19.8\% vs. $40.4 \%$ with no experience) were significantly less likely to agree that they "would not want to know if I have cancer."

\section{Discussion}

In this population-based sample of US adults, there was high agreement (>80\%) with the positively framed statements about cancer outcomes and the value of timely help seeking for a potential cancer symptom. Moreover, there were few differences in these positive beliefs based on SES or sociodemographic factors. On the other hand, there was lower agreement with the negatively framed statements among US adults, and greater variation especially by education level such that those with lower education were more likely to endorse each negative cancer belief in the adjusted models.

Previous studies using the ABC survey to examine associations between SES and positive and negative cancer beliefs in the $\mathrm{UK}^{20}$ and Denmark ${ }^{22}$ also found that most adults agreed with positive cancer beliefs and that few sociodemographic differences were evident. For example, $94.6 \%$ of US adults agreed that "going to the doctor as quickly as possible after noticing a symptom of cancer could increase the chances of surviving," while $97.6 \%$ and $97.2 \%$ of adults from the UK and Denmark agreed with this statement, respectively. Taken together, these results suggest that across countries with differing healthcare systems, beliefs about 
cancer's limited effect on daily living, the value of early diagnosis, and the curability of cancer are generally held across different SES groups. In turn, these results suggest that SES differences in negative cancer beliefs are likely to be found across variable healthcare systems, and that strategies used in other countries to help combat negative cancer beliefs may also be applicable and useful in the US, despite differences in the structure of healthcare systems.

Also similar to previous $\mathrm{ABC}$ studies, lower education consistently predicted higher odds of endorsing negative cancer beliefs in the present study: respondents with a high school education or less had two to three times higher odds of endorsing each of the negative beliefs. There are many potential explanations for these associations. Individuals in lower SES groups tend to have lower health literacy, which could result in lower understanding of and exposure to scientific information about improvements in cancer outcomes, which are frequently disseminated in ways better-suited to those with higher literacy. ${ }^{16,25}$ However, we might also then expect to observe similar SES differences in endorsement of positively framed beliefs, which we did not observe. Lower SES groups may also find negatively framed items harder to interpret and, in turn, be more likely to agree with negative statements in general; however, the $\mathrm{ABC}$ measure does not contain non-cancer negatively framed items that could be used to examine this hypothesis. Alternatively, lower SES groups may have more experience with poorer cancer outcomes within their close and wider social networks and, as a result, have more negative expectations about cancer outcomes. In the current sample, people with cancer experience were more likely to want to know if they have cancer, a finding also reported in the $\mathrm{UK}^{20}$ and in Denmark, ${ }^{29}$ yet less likely to believe people with cancer can continue with their normal activities. This finding may reflect that people with cancer experience value complete knowledge about having cancer, perhaps due to perceiving a more proactive approach to 
managing cancer as more effective. However, cancer experience did not significantly differ among education groups and was not an independent predictor of agreement for two of the negative statements. The cancer experience item used in the ABC measure does not ask about the breadth or quality of cancer experiences, and a more nuanced measure of cancer experience may show different associations with beliefs. In addition to educational attainment, other social determinants of health, such as access to quality healthcare, likely influence the development of negative cancer beliefs. Future research should explore how social determinants work together to shape the formation of cancer beliefs.

The proportions of adults agreeing with positive and negative beliefs were similar across the countries. For example, agreement with "These days, many people with cancer can expect to continue with normal activities and responsibilities" was $83.9 \%, 88.0 \%$, and $83.3 \%$ in the US, UK, and Denmark, respectively. One notable exception to this similarity was agreement with "I would not want to know if I had cancer," which was markedly higher in the US $(23.3 \%)$ than in either England (11.7\%) or Denmark (10.0\%). It is unclear why US adults may be more avoidant of cancer diagnosis information, particularly since beliefs about the curability of cancer were high. It is possible that beliefs about the potential negative implications of a cancer diagnosis (e.g., financial burden and stigma of a diagnosis) may be greater in the US due to a lack of universal healthcare and, in turn, encourage avoidance among certain populations. This possibility is concerning, since cancer avoidance may discourage the early detection behaviors that could result in an earlier stage at diagnosis and better cancer outcomes. Future research should focus on better understanding the basis of and contributors to this belief that may explain differences in cancer survival among developed countries. 
Similar to previous studies from the US, which have relied on items from HINTS, we also found SES differences in negative cancer beliefs. ${ }^{11,16,23-26}$ Though HINTS is also a population-based survey of US adults, the cancer beliefs items are distinct, and results for similar items provide different conclusions. In particular, one item from HINTS that has appeared on several cycles $(2008,2013$, and 2017) is "When I think of cancer, I automatically think of death." Across time, more than 50\% of US adults have agreed with this statement. For example, one study that examined this belief in HINTS 2008 reported that $61 \%$ of US adults agreed with the statement, ${ }^{10}$ a proportion that was also reported in the 2017 cycle. ${ }^{30}$ This proportion contrasts with our finding that $26.7 \%$ of adults agreed with a similar item on the $\mathrm{ABC}$ measure ("A diagnosis of cancer is a death sentence"). Disparate results may be explained by differences in the sampled population (e.g., age groups) and unique aspects of the item wording that influence the response; however, the results suggest that more research is needed to better understand how people are interpreting similarly worded items about associations between cancer and death. In addition, there are important design implications for attitudinal population surveys. Future studies should aim to measure both positively and negatively framed beliefs, since endorsing more negative cancer beliefs is not equivalent to endorsing fewer positive cancer beliefs, and both types of beliefs clearly coexist.

\section{Study Limitations}

The current study had several strengths including the large, nationally representative sample and the use of a validated survey instrument that permitted cross-country comparison. In addition, the inclusion of both positive and negative cancer beliefs is unique to the $\mathrm{ABC}$ measure. Several limitations also warrant mention. Since this was a cross-sectional study, we are unable to assess how associations between SES and beliefs are formed over time or whether 
current beliefs are predictive of future early detection behaviors. Furthermore, objectively observed early detection behaviors were not included in the $\mathrm{ABC}$ measure. More research is needed to assess whether positive and negative beliefs about cancer are predictive of these behaviors. The use of education as a proxy for SES may be seen as a limitation; however, given the older age of the sample, education is more appropriate than income and employment status, which might not be as accurate. ${ }^{31}$

\section{Clinical Implications}

Our results suggest that interventions to improve early cancer detection behaviors targeting lower SES groups may benefit from focusing on reducing negative beliefs, rather than increasing positive beliefs. In addition, our findings match those from other countries that administered the same survey, suggesting that SES differences in negative cancer beliefs are independent of healthcare system structure and that strategies used in other countries to reduce negative cancer beliefs may be useful in the US.

\section{Conclusions}

US adults generally agreed with positive statements about the curability of cancer, value of early diagnosis, and ability to function post-cancer diagnosis; however, adults with lower education were more likely to agree with negative statements. A better understanding of the mechanisms through which lower SES contributes to both negative and more mixed beliefs about cancer outcomes is needed. In addition, more work is needed to establish whether and how both positive and negative beliefs interact to influence cancer detection behaviors and can be thusly addressed to improve uptake of these behaviors in lower SES groups. 
Acknowledgements: The opinions expressed by the authors are their own and this material should not be interpreted as representing the official viewpoint of the U.S. Department of Health and Human Services, the National Institutes of Health or the National Cancer Institute.

Conflict of Interest: The authors declare no potential conflicts of interest.

Data Availability Statement: The data that support the findings of this study are available from the corresponding author upon reasonable request. 


\section{References}

1. Siegel RL, Miller KD, Jemal A. Cancer statistics, 2019. CA Cancer J Clin. 2019;69(1):734.

2. Byers TE, Wolf HJ, Bauer KR, et al. The impact of socioeconomic status on survival after cancer in the United States: Findings from the National Program of Cancer Registries Patterns of Care Study. Cancer. 2008;113(3):582-591.

3. Scott SE, Walter FM, Webster A, Sutton S, Emery J. The model of pathways to treatment: Conceptualization and integration with existing theory. Br J Health Psychol. $2013 ; 18(1): 45-65$.

4. World Health Organization. Guide to cancer early diagnosis. Geneva, Switzerland: World Health Organization; 2017.

5. White A, Thompson TD, White MC, et al. Cancer screening test use - United States, 2015. Morb Mortal Wkly Rep. 2017;66(8):201-206.

6. Rendle KA, Sarma EA, Quaife S, et al. Cancer symptom recognition and anticipated delays in seeking care among U.S. adults. Am J Prev Med. 2019;57(1):e1-9.

7. Quaife SL, Forbes LJ, Ramirez AJ, et al. Recognition of cancer warning signs and anticipated delay in help-seeking in a population sample of adults in the UK. Br J Cancer. 2014;110(1):12-18.

8. Pedersen AF, Forbes L, Brain K, et al. Negative cancer beliefs, recognition of cancer symptoms and anticipated time to help-seeking: an international cancer benchmarking partnership (ICBP) study. BMC Cancer. 2018;18(1):363. 
9. Forbes LJ, Warburton F, Richards MA, Ramirez AJ. Risk factors for delay in symptomatic presentation: A survey of cancer patients. Br J Cancer. 2014;111(3):581588.

10. Moser RP, Arndt J, Han PK, Waters EA, Amsellem M, Hesse BW. Perceptions of cancer as a death sentence: Prevalence and consequences. J Health Psychol. 2014;19(12):15181524.

11. Emanuel AS, Kiviniemi MT, Howell JL, et al. Avoiding cancer risk information. Soc Sci Med. 2015;147:113-120.

12. McCutchan GM, Wood F, Edwards A, Richards R, Brain KE. Influences of cancer symptom knowledge, beliefs and barriers on cancer symptom presentation in relation to socioeconomic deprivation: A systematic review. BMC Cancer. 2015;15:1000.

13. von Wagner C, Good A, Whitaker KL, Wardle J. Psychosocial determinants of socioeconomic inequalities in cancer screening participation: a conceptual framework. Epidemiol Rev. 2011;33:135-147.

14. Powe BD, Finnie R. Cancer fatalism: The state of the science. Cancer Nurs. $2003 ; 26(6): 454-465$.

15. Beeken RJ, Simon AE, von Wagner C, Whitaker KL, Wardle J. Cancer fatalism: Deterring early presentation and increasing social inequalities? Cancer Epidemiol Biomarkers Prev. 2011;20(10):2127-2131.

16. Kobayashi LC, Smith SG. Cancer fatalism, literacy, and cancer information seeking in the American public. Health Educ Behav. 2016;43(4):461-470. 
17. Kowalkowski MA, Hart SL, Du XL, Baraniuk S, Latini DM. Cancer perceptions: Implications from the 2007 Health Information National Trends Survey. J Cancer Surviv. 2012;6(3):287-295.

18. Niederdeppe J, Levy AG. Fatalistic beliefs about cancer prevention and three prevention behaviors. Cancer Epidemiol Biomarkers Prev. 2007;16(5):998-1003.

19. Lyratzopoulos G, Liu MP, Abel GA, Wardle J, Keating NL. The association between fatalistic beliefs and late stage at diagnosis of lung and colorectal cancer. Cancer Epidemiol Biomarkers Prev. 2015;24(4):720-726.

20. Quaife SL, Winstanley K, Robb KA, et al. Socioeconomic inequalities in attitudes towards cancer: An international cancer benchmarking partnership study. Eur J Cancer Prev. 2015;24(3):253-260.

21. Simon AE, Forbes LJ, Boniface D, et al. An international measure of awareness and beliefs about cancer: Development and testing of the ABC. BMJ Open. 2012;2(6):e001758.

22. Hvidberg L, Wulff CN, Pedersen AF, Vedsted P. Barriers to healthcare seeking, beliefs about cancer and the role of socio-economic position. A Danish population-based study. Prev Med. 2015;71:107-113.

23. Assari S, Khoshpouri P, Chalian H. Combined effects of race and socioeconomic status on cancer beliefs, cognitions, and emotions. Healthcare (Basel). 2019;7(1):17.

24. Cunningham SA, Yu R, Shih T, et al. Cancer-related risk perceptions and beliefs in Texas: Findings from a 2018 population-level survey. Cancer Epidemiol Biomarkers Prev. 2019;28(3):486-494. 
25. Fleary SA, Paasche-Orlow MK, Joseph P, Freund KM. The relationship between health literacy, cancer prevention beliefs, and cancer prevention behaviors. J Cancer Educ. 2019;34(5):958-965.

26. Vanderpool RC, Huang B, Deng Y, et al. Cancer-related beliefs and perceptions in Appalachia: Findings from 3 states. J Rural Health. 2019;35(2):176-188.

27. Forbes LJ, Simon AE, Warburton F, et al. Differences in cancer awareness and beliefs between Australia, Canada, Denmark, Norway, Sweden and the UK (the International Cancer Benchmarking Partnership): Do they contribute to differences in cancer survival? Br J Cancer. 2013;108(2):292-300.

28. Sarma EA, Rendle KA, Kobrin SC. Cancer symptom awareness in the US: Sociodemographic differences in a population-based survey of adults. Prev Med. 2020;132:106005.

29. Pedersen AF, Vedsted P. Cancer beliefs in cancer survivors, cancer relatives and persons with no cancer experience. Scand J Public Health. 2019;47(5):497-503.

30. National Cancer Institute. Health Information National Trends Survey. Available from: https://hints.cancer.gov/view-questions-topics/questiondetails.aspx?PK_Cycle=3\&qid=509 [Accessed 30 June 2020].

31. Grundy E, Holt G. The socioeconomic status of older adults: How should we measure it in studies of health inequalities? J Epidemiol Community Health. 2001;55(12):895-904. 
Table 1. Sample Characteristics $(N=1,425)$

\begin{tabular}{lcc}
\hline Variable & $\mathrm{n}$ & $\%$ \\
\hline Gender & & \\
$\quad$ Women & 942 & 53.5 \\
$\quad$ Men & 483 & 46.5 \\
Age & & \\
$\quad$ 50-64y & 687 & 56.0 \\
$\quad$ 65-99y & 736 & 44.0 \\
Marital status & & \\
$\quad$ Married/cohabiting & 726 & 62.8 \\
$\quad$ Single/divorced/widowed & 684 & 37.2 \\
Race & & \\
$\quad$ White & 1185 & 79.2 \\
$\quad$ Non-White & 211 & 20.8 \\
Education & & \\
$\quad$ Bachelors or above & 691 & 30.7 \\
$\quad$ Some college & 445 & 23.8 \\
$\quad$ High school or lower & 289 & 45.5 \\
Self-rated health & & \\
$\quad$ Good & 1065 & 71.0 \\
$\quad$ Fair/poor & 354 & 29.1 \\
Cancer experience & & \\
$\quad$ None \\
$\quad$ Self/close other & 235 & 17.5 \\
Totals may not sum & 1181 & 82.5 \\
\hline
\end{tabular}

Totals may not sum due to missing data. Percentages weighted for survey design. 
Table 2. Multivariable Logistic Regression Models Predicting Agreement with Positively Framed Cancer Beliefs

\begin{tabular}{|c|c|c|c|c|c|c|c|c|c|}
\hline & \multicolumn{3}{|c|}{$\begin{array}{l}\text { These days, many people with } \\
\text { cancer can expect to continue } \\
\text { with normal activities and } \\
\text { responsibilities } \\
\qquad(N=1410)\end{array}$} & \multicolumn{3}{|c|}{ Cancer can often be cured } & \multicolumn{3}{|c|}{$\begin{array}{l}\text { Going to the doctor as quickly as } \\
\text { possible after noticing a } \\
\text { symptom of cancer could } \\
\text { increase the chances of surviving } \\
\qquad(N=1420)\end{array}$} \\
\hline & $\mathrm{n}(\%)$ & $\mathrm{aOR}$ & $99 \% \mathrm{CI}$ & $\mathrm{n}(\%)$ & $\mathrm{aOR}$ & $99 \% \mathrm{CI}$ & $\mathrm{n}(\%)$ & $\mathrm{aOR}$ & $99 \% \mathrm{CI}$ \\
\hline Total sample & $1200(83.9)$ & & & $1172(82.8)$ & & & $1359(94.6)$ & & \\
\hline \multicolumn{10}{|l|}{ Gender } \\
\hline Men & $412(84.2)$ & 1.00 & - & $407(85.8)$ & 1.00 & - & $459(94.0)$ & 1.00 & - \\
\hline Women & $788(83.6)$ & 1.11 & $0.61-2.03$ & $765(80.2)$ & 0.65 & $0.37-1.13$ & $900(95.1)$ & 1.26 & $0.44-3.63$ \\
\hline \multicolumn{10}{|l|}{ Age group } \\
\hline $50-64 y$ & $579(83.3)$ & 1.00 & - & $549(80.6)$ & 1.00 & - & $651(94.1)$ & 1.00 & - \\
\hline $65-99 y$ & $620(84.6)$ & 0.93 & $0.53-1.64$ & $622(85.7)$ & 0.68 & $0.40-1.51$ & 707 (95.2) & 0.70 & $0.27-1.81$ \\
\hline \multicolumn{10}{|l|}{ Marital status } \\
\hline Married/cohabiting & $623(84.5)$ & 1.00 & - & $616(84.9)$ & 1.00 & - & 695 (95.9) & 1.00 & - \\
\hline Single/divorced/widowed & $565(82.9)$ & 0.95 & $0.55-1.65$ & $543(79.4)$ & 0.75 & $0.44-1.27$ & $652(92.8)$ & 0.57 & $0.19-1.69$ \\
\hline \multicolumn{10}{|l|}{ Race } \\
\hline White & $1008(84.7)$ & 1.00 & - & $990(83.7)$ & 1.00 & - & $1129(94.5)$ & 1.00 & - \\
\hline Non-White & $169(80.5)$ & 0.85 & $0.39-1.87$ & 159 (78.6) & 0.85 & $0.42-1.73$ & $202(94.7)$ & 1.17 & $0.31-4.47$ \\
\hline \multicolumn{10}{|l|}{ Education } \\
\hline Bachelor's or above & 597 (87.9) & 1.00 & - & $587(85.1)$ & 1.00 & - & $674(95.8)$ & 1.00 & - \\
\hline Some college & $373(83.3)$ & 0.71 & $0.38-1.32$ & $362(82.5)$ & 0.92 & $0.51-1.64$ & $417(94.0)$ & 0.75 & $0.21-2.71$ \\
\hline High school or lower & $230(81.5)$ & 0.66 & $0.34-1.27$ & $223(81.5)$ & 0.89 & $0.50-1.58$ & $268(94.0)$ & 0.82 & $0.21-3.25$ \\
\hline \multicolumn{10}{|l|}{ Self-rated health } \\
\hline Good & $922(87.8)$ & 1.00 & - & $896(85.2)$ & 1.00 & - & $1019(94.8)$ & 1.00 & - \\
\hline Fair/poor & $274(75.0)$ & 0.44 & $0.24-0.82$ & $270(76.8)$ & 0.60 & $0.33-1.06$ & 334 (93.9) & 0.89 & $0.30-2.60$ \\
\hline \multicolumn{10}{|l|}{ Cancer experience } \\
\hline None & 205 (91.6) & 1.00 & - & $191(79.8)$ & 1.00 & - & $222(91.0)$ & 1.00 & — \\
\hline Self/close other & 988 (82.3) & 0.38 & $0.16-0.91$ & 974 (83.4) & 1.30 & $0.65-2.60$ & $1128(95.3)$ & 1.53 & $0.47-5.01$ \\
\hline
\end{tabular}

Abbreviations: aOR, adjusted odds ratio; $\mathrm{CI}$, confidence interval. 
Table 3. Multivariable Logistic Regression Models Predicting Agreement with Negatively Framed Cancer Beliefs

\begin{tabular}{|c|c|c|c|c|c|c|c|c|c|}
\hline & \multicolumn{3}{|c|}{$\begin{array}{l}\text { A diagnosis of cancer is a death } \\
\text { sentence }\end{array}$} & \multicolumn{3}{|c|}{$\begin{array}{l}\text { I would not want to know if I } \\
\text { have cancer }\end{array}$} & \multicolumn{3}{|c|}{$\begin{array}{l}\text { Most cancer treatment is worse } \\
\text { than the cancer itself }\end{array}$} \\
\hline & \multicolumn{3}{|c|}{$(N=1410)$} & \multicolumn{3}{|c|}{$(N=1397)$} & \multicolumn{3}{|c|}{$(N=1411)$} \\
\hline & $\mathrm{n}(\%)$ & $\mathrm{aOR}$ & $99 \% \mathrm{CI}$ & $\mathrm{n}(\%)$ & $\mathrm{aOR}$ & $99 \% \mathrm{CI}$ & $\mathrm{n}(\%)$ & $\mathrm{aOR}$ & $99 \% \mathrm{CI}$ \\
\hline Total sample & $334(26.7)$ & & & $255(23.3)$ & & & $772(59.6)$ & & \\
\hline \multicolumn{10}{|l|}{ Gender } \\
\hline Men & $120(28.6)$ & 1.00 & - & $95(26.1)$ & 1.00 & - & $241(56.3)$ & 1.00 & - \\
\hline Women & $214(25.0)$ & 0.75 & $0.45-1.25$ & $160(20.9)$ & 0.64 & $0.37-1.10$ & $531(62.4)$ & 1.14 & $0.75-1.74$ \\
\hline \multicolumn{10}{|l|}{ Age group } \\
\hline $50-64 y$ & $177(27.6)$ & 1.00 & - & $129(22.7)$ & 1.00 & - & $397(62.2)$ & 1.00 & - \\
\hline $65-99 y$ & $156(25.4)$ & 1.27 & $0.78-2.06$ & $125(24.0)$ & 0.89 & $0.52-1.53$ & $373(56.2)$ & 1.38 & $0.92-2.06$ \\
\hline \multicolumn{10}{|l|}{ Marital status } \\
\hline Married/cohabiting & $149(25.0)$ & 1.00 & - & $114(19.7)$ & 1.00 & - & $368(57.0)$ & 1.00 & - \\
\hline Single/divorced/widowed & $178(28.7)$ & 1.21 & $0.75-1.97$ & $137(28.9)$ & 1.37 & $0.79-2.36$ & $393(63.5)$ & 1.22 & $0.81-1.85$ \\
\hline \multicolumn{10}{|l|}{ Race } \\
\hline White & $280(27.0)$ & 1.00 & 一 & $191(20.7)$ & 1.00 & 一 & $628(58.3)$ & 1.00 & 一 \\
\hline Non-White & $47(25.4)$ & 0.89 & $0.44-1.78$ & $56(33.3)$ & 1.87 & $0.95-3.68$ & $127(63.7)$ & 1.15 & $0.64-2.07$ \\
\hline \multicolumn{10}{|l|}{ Education } \\
\hline Bachelor's or above & $123(17.4)$ & 1.00 & 一 & $79(15.7)$ & 1.00 & 一 & $319(45.2)$ & 1.00 & 一 \\
\hline Some college & $117(25.8)$ & 1.72 & $1.03-2.87$ & $81(17.1)$ & 1.20 & $0.63-2.28$ & $266(61.4)$ & 1.79 & $1.18-2.71$ \\
\hline High school or lower & $94(33.2)$ & 2.51 & $1.45-4.37$ & $95(31.6)$ & 2.88 & $1.54-5.36$ & $187(68.2)$ & 2.43 & $1.50-3.94$ \\
\hline \multicolumn{10}{|l|}{ Self-rated health } \\
\hline Good & $223(24.4)$ & 1.00 & - & $173(21.1)$ & 1.00 & - & $560(57.2)$ & 1.00 & - \\
\hline Fair/poor & $110(32.3)$ & 1.43 & $0.85-2.41$ & $82(28.8)$ & 1.26 & $0.71-2.26$ & $209(65.5)$ & 1.23 & $0.77-1.97$ \\
\hline \multicolumn{10}{|l|}{ Cancer experience } \\
\hline None & $53(22.3)$ & 1.00 & - & $67(40.4)$ & 1.00 & - & $129(59.3)$ & 1.00 & - \\
\hline Self/close other & $278(27.6)$ & 1.48 & $0.76-2.88$ & $185(19.8)$ & 0.42 & $0.14-0.72$ & $637(59.5)$ & 1.04 & $0.59-1.86$ \\
\hline
\end{tabular}

Abbreviations: aOR, adjusted odds ratio; CI, confidence interval. 